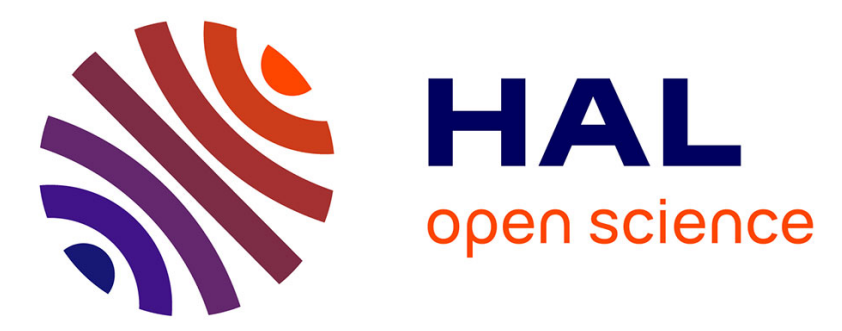

\title{
A 2D Transducer Structure for the Excitation of Surface Acoustic Wave
}

\author{
W. Daniau, T. Baron, J. Garcia, Thierry Laroche, S. Ballandras
}

\section{To cite this version:}

W. Daniau, T. Baron, J. Garcia, Thierry Laroche, S. Ballandras. A 2D Transducer Structure for the Excitation of Surface Acoustic Wave. 24th European Frequency and Time Forum (EFTF 2010), Apr 2010, Noordwijk, Netherlands. 10.1109/EFTF.2010.6533656 . hal-00578957

\section{HAL Id: hal-00578957 https://hal.science/hal-00578957}

Submitted on 14 May 2021

HAL is a multi-disciplinary open access archive for the deposit and dissemination of scientific research documents, whether they are published or not. The documents may come from teaching and research institutions in France or abroad, or from public or private research centers.
L'archive ouverte pluridisciplinaire HAL, est destinée au dépôt et à la diffusion de documents scientifiques de niveau recherche, publiés ou non, émanant des établissements d'enseignement et de recherche français ou étrangers, des laboratoires publics ou privés. 


\title{
A 2D Transducer Structure for the Excitation of Surface Acoustic Wave
}

\author{
$24^{\text {th }}$ European Frequency and time Forum \\ 13-16 April 2010
}

\author{
William Daniau, Thomas Baron, Julien Garcia, Thierry Laroche, Sylvain Ballandras \\ ${ }^{(1)}$ FEMTO-ST, CNRS-UFC-ENSMM-UTBM UMR 6174 , \\ 32 Avenue de l'Observatoire, 25044 Besançon, FRANCE \\ Email: william.daniau@femto-st.fr
}

\section{INTRODUCTION}

The standard fabrication of surface acoustic wave devices exploiting inter-digital transducers (IDTs) on single crystal substrates is based on metal deposition and patterning using photolithography technologies. It has been demonstrated these passed years that thin film based devices also could answer the demand for radio-frequency (RF) passive devices (filters, sources) for signal treatment. Varieties of new transducers and wave guides have been developed and implemented for the fabrication of high frequency devices exhibiting wave characteristics comparable to those of standard surface acoustic waves on lithium tantalite, the most used material for passive low loss RF filters. Most of these devices are built using the celebrated inter-digited transducer invented by White and Voltmer [1] consisting in a 1D periodic grating of electrodes driven with an alternated $+\mathrm{V} /-\mathrm{V}$ excitation voltage.

In this work, we investigate the possibility to exploit a 2D periodic transducer consisting of square dots or pads exhibiting a double excitation potential alternation (along $x_{1}$ and $x_{2}$, here the axes defining the surface). We first briefly recall the simulation principle of such devices (based on coupled finite element analysis/boundary element method - FEA/BEM) and report preliminary results under the form of harmonic admittance for such transducer deposited on quartz or lithium tantalate or lithium niobate and we point out the main difference between these various situations. These computations allows us to identifiy standard surface waves exhibiting a plane wave front (more or less colinear to a propagation direction) and waves exhibiting complex surface vibration that could be understood as an ellipsoidal polarization.

We then propose an implementation of the transducer on lithium niobate, exploiting the strong coupling of Rayleigh Surface Acoustic Waves (SAW) on $\mathrm{LiNbO}_{3}(\mathrm{YXl}) / 128^{\circ}$ and taking advantage of the strong dielectric properties of such substrate for achieving the pad interconnection. Hence, a combinaison of passivation pads and aluminum metallization allows for connecting square individual electrodes one another as expected theoretically. Characterization of experimental devices is reported. The agreement between theory and measurements and the quality of the admittance curves allow for identifying the excited contributions and provides a first demonstration of the possibility to excite spherically polarized waves using such transducer structures.

\section{FEA/BIM ANALYSIS}

Figure 1 shows a general scheme of the considered periodic device geometry. Periodicities are assumed taking place in the plane $\left(x_{1}, x_{2}\right)$. The corresponding periods are noted $d_{1}$ and $d_{2}$. The transducer is assumed inhomogeneous along $x_{3}$, imposing to mesh the corresponding region. It can be composed of various materials with arbitrary shapes assuming they can be well represented using an elastic displacement (and if required electrical potential) based FEA formulation. Material losses can also be considered by assuming complex elastic, piezoelectric and dielectric coefficients [2]. Assuming infinitely periodic strustures along both axes allows for generalizing the concept of harmonic excitation which is intensively used for the analysis of 1D-periodic structures such as SAW wave guides [3]. We then consider that the excitation potential of a cell (one period) indexed by $n$ and $m$ is related to a reference cell potential $\varphi 0$ via excitation parameters $\gamma_{1}$ and $\gamma_{2}$ lying respectively along $x_{1}$ and $x_{2}$ [4]. 


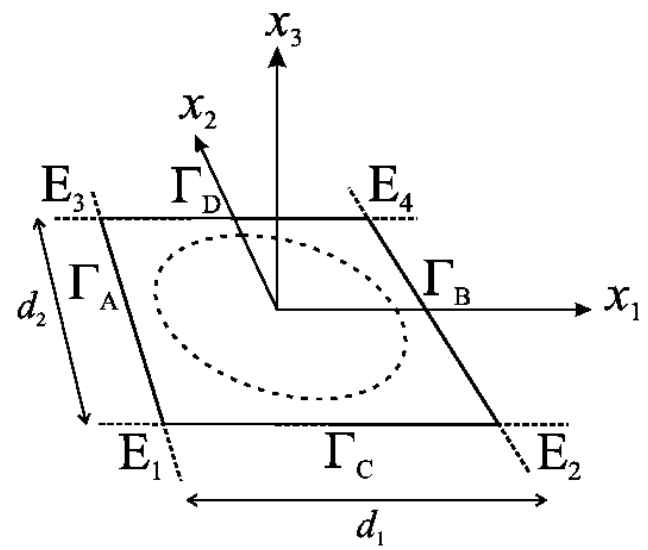

Fig.1 Axes and notation conventions used to represent a 2D periodic structure

The basic equations governing periodic FEA computations are now briefly recalled. As already reported in previous references [4,5], FEA can be performed for periodic devices with rather simple modifications of the basic algebraic formula relating the displacement and electrical fields to the boundary solicitations. In the case of 2D periodicity devices, it first consists in relating all the degrees of freedom (dof) on boundaries in regards, i.e. $\Gamma_{A}$ with $\Gamma_{B}$ and $\Gamma_{C}$ with $\Gamma_{D}$, and those on the corners of the mesh noted $\mathrm{E}_{1}$ to $\mathrm{E}_{4}$, yielding the following expressions first proposed in ref. [5]

$$
\begin{aligned}
& u_{\Gamma_{B}}=u_{\Gamma_{A}} e^{-j \sqsubset \pi \gamma} \\
& u_{\Gamma_{D}}=u_{\Gamma_{C}} e^{-j[\pi \gamma} \\
& u_{E_{\curlyvee}}=u_{E_{\square}} e^{-j\left\lceil\pi \gamma_{\square}\right.} \\
& u_{E_{r}}=u_{E} e^{-j \llbracket \pi \gamma} \\
& \left.u_{E_{\varepsilon}}=u_{E_{\square}} e^{-j \llbracket \pi\left(\gamma_{1}+\gamma\right.}\right)
\end{aligned}
$$

in which $u$ holds for the generalized displacements (including the electrical potential), and $\gamma_{1}$ and $\gamma_{r}$ are the abovedefined excitation parameters. This relation then is used to reduce the number of independent dof of the FEA model. This is performed without changing the total number of dof of the problem, simply by using a variable change operator $C$ that depends on the excitation parameter $\gamma$. Note that relation (4) does not depend on the kind of FEA performed and can be applied for linear analysis in harmonic, modal and transient regimes as well [5]. For harmonic analyses, the FEA system to solve reads

$$
{ }^{t}\left[C\left(\gamma_{1}, \gamma_{\square}\right)\right]\left[K-\omega^{r} M-X(\omega)\right]\left[C\left(\gamma_{1}, \gamma_{\square}\right)\right]\{v\}={ }^{t}\left[C\left(\gamma_{1}, \gamma_{\square}\right)\right]\{F\}
$$

where $K$ is the stiffness matrix, $M$ is the mass matrix, $X$ is the frequency-dependent radiation matrix corresponding to BEM, $F$ is the external solicitation vector (Neumann boundary conditions) and $v$ is the new set of independent dof. The operator $C$ is a triangular complex matrix. Due to the presence of radiation boundary conditions and in the case $K$ is complex (i.e. accounting for material losses), the matrix product in (2) results in a general complex matrix with no particular properties. In the piezoelectric case, those matrices are generalized (i.e. accounting also for the electrostatic energy equilibrium) and $M$ then is singular. This approach is naturally well suited for harmonic analysis, solving the algebraic system (2) for each frequency and excitation parameter values.We particularly focused on one excitation configuration consisting in potential alternation along both period $d_{1}$ and $d_{2}$ as illustrated in fig.2. This yields a kind of triangular symmetry atop the surface and is expected to favour the excitation of wave with complex polarization, as we intend to check the possibility to create such propagation conditions. It is however clear that the actual implementation of such an excitation configuration is quite difficult to achieve, as we can not imagine the fabrication of metal bridges for accessing all the square dots of the surface as proposed long time ago for the first single phase unidirectionnal transducers [6]. We then imagine the use of a dielectric spacer allowing for preventing the presence of electrical charge at the surface between the dots but allowing for the 
connection we are looking for. This is graphically illustrated in fig.3, showing an example of mesh in which the excitation structure is decomposed to illustrate the proposed approach.

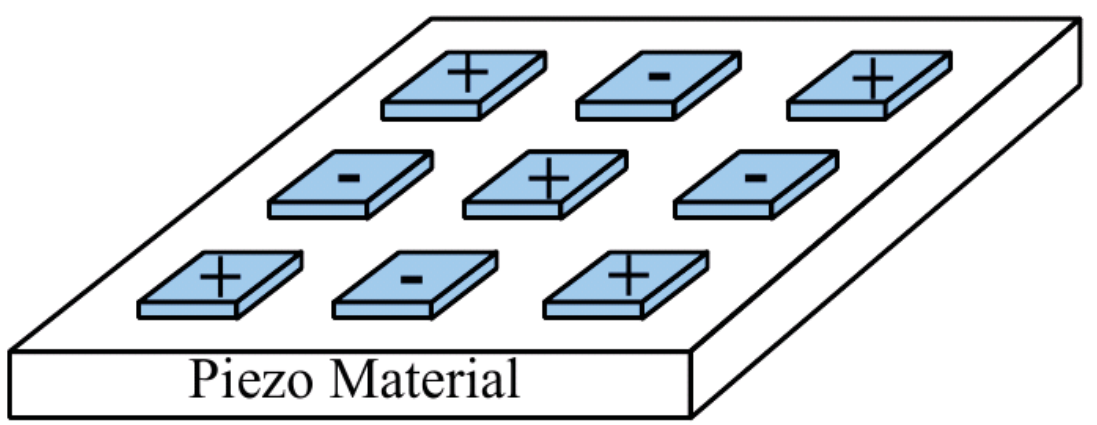

Fig.2 A 2D excitation structure based on a double potential alternation to promote the excitation of surface waves exhibiting complex polarization (the $2 \mathrm{D}$ equivalent of the usual interdigital transducer)

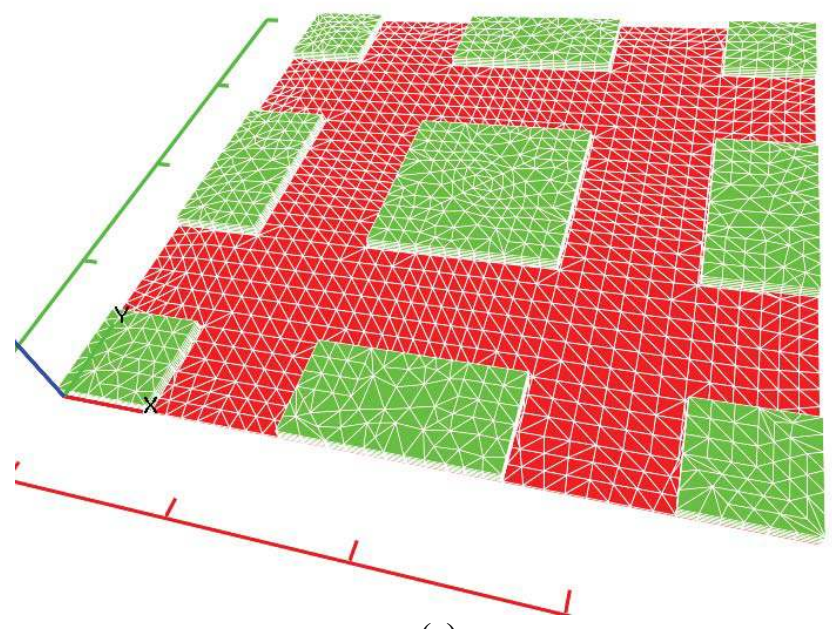

(a)

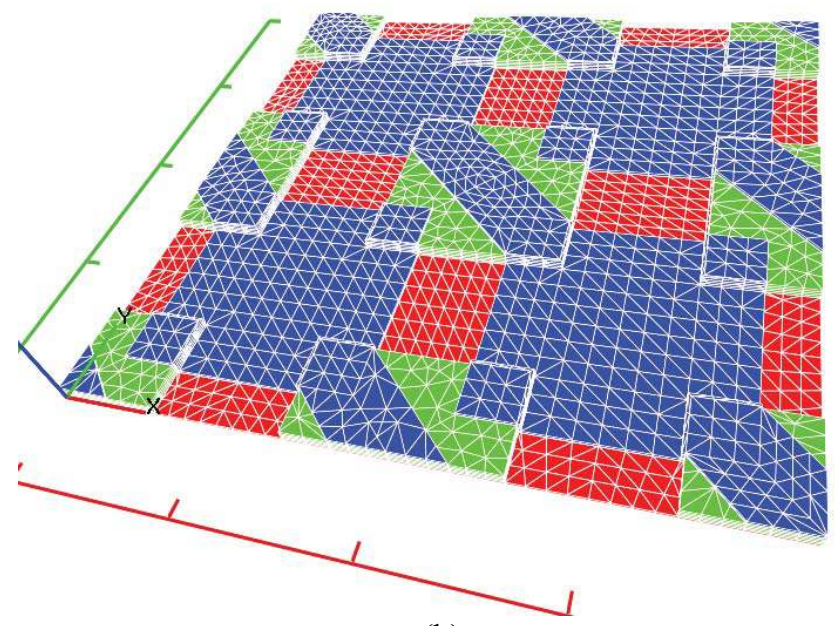

(b)

Fig.3 An example of simplified 3D mesh used to simulate the 2D periodic transducer, numbers 1 and 2 represents the two electrode polarities, references 3 to 10 are used to imposed periodic boundary conditions, the period of the grating is set to $25 \mu \mathrm{m}$ (a) localization of the dielectric - $\mathrm{SiO}_{2}$ - spacers, the green square dots (b) deposition of the $\mathrm{Al}$ electrodes above the structure shown in (a), the blue strips and squares

This kind of mesh has been used for the computation of the harmonic admittance of an infinite 2D periodic transducer according to the excitation conditions of fig.2. We then simply apply opposite potentials to the adjacent strips and set the two excitation parameters to an integer value (typically one). We particularly focused our interest on $\mathrm{LiNbO}_{3}$ and more specifically the (YXl)/128 cut because of its advantageous electromechanical coupling (and its availability), although we also tested this configuration with AT cut of quartz (this point is discussed later). Figure 4 shows the harmonic admittance obtained along this approach. This curve reveals the excistence of two surface modes and a quite complex behavior of the conductance, indicating a plurality of mode radiated from the surface and/or partially guided by the grating (particularly shown by fig.4a). To better understand the operation of the transducer, we have plotted the deformed shape of the surface for the two guided modes in fig. $5 \mathrm{a} \& \mathrm{~b}$. It turns out that the propagation takes place in both case along the median trace of the dielectric dots. In that case, the wavelength is then equal to $35.5 \mu \mathrm{m}$, yielding the observed contributions near $100 \mathrm{MHz}$. In this configuration, the transducer does not really allow for exciting complex propagation polarization but behaves more like a natural partially directionnal transducer (a small resonance at the beginning of the stop band but a large one at its end). However, analysis of higher order contributions (SSBW, spurious modes above $200 \mathrm{MHz}$ ) shows more complex surface vibration, but we do not report this here as there at this time no idea on how exploiting those phenomena. 


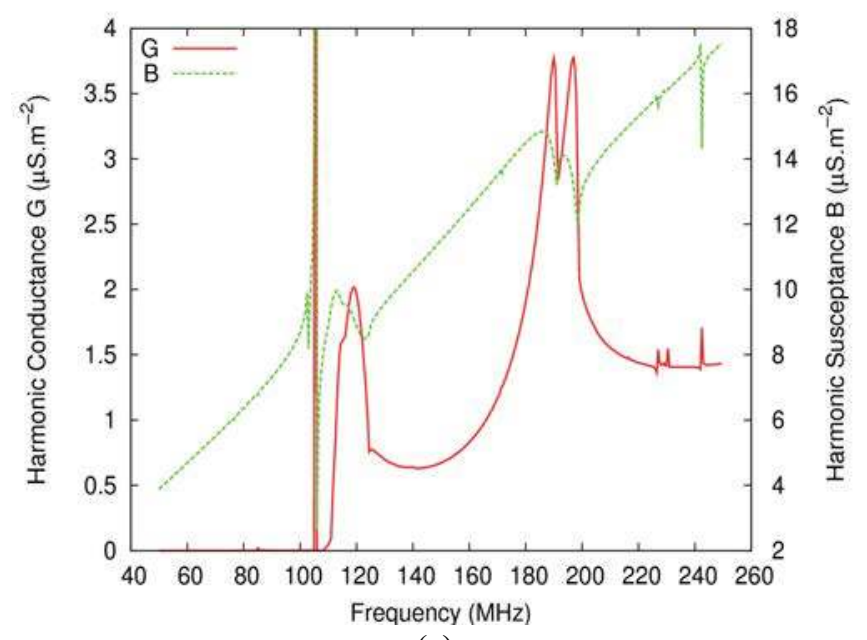

(a)

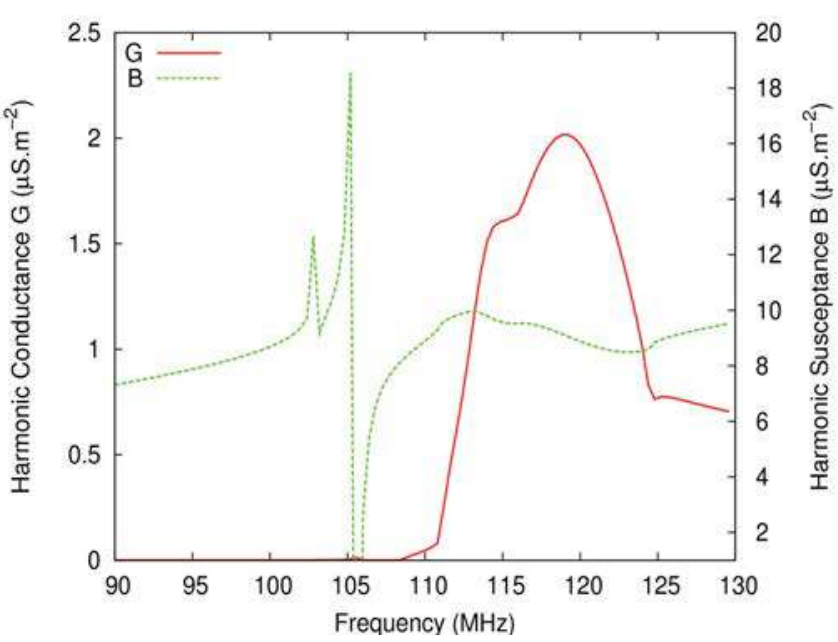

(b)

Fig.4 Harmonic admittance of the infinite 2D periodic grating of fig.3 (a) wide spectrum plot (b) focusing on the guided modes and SSBW contributions

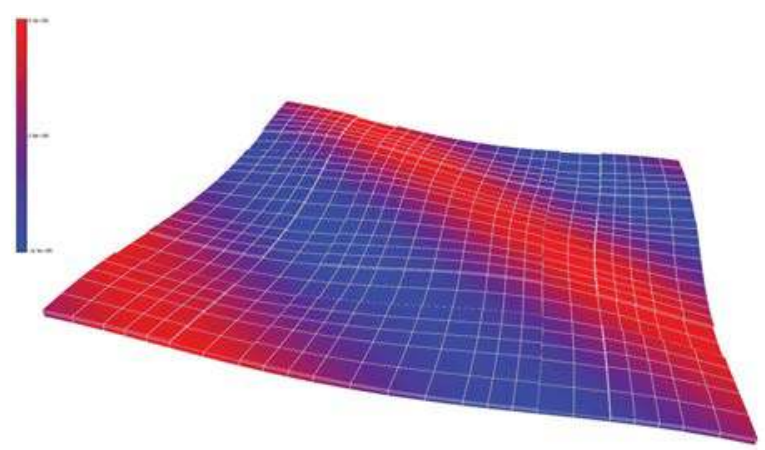

(a)

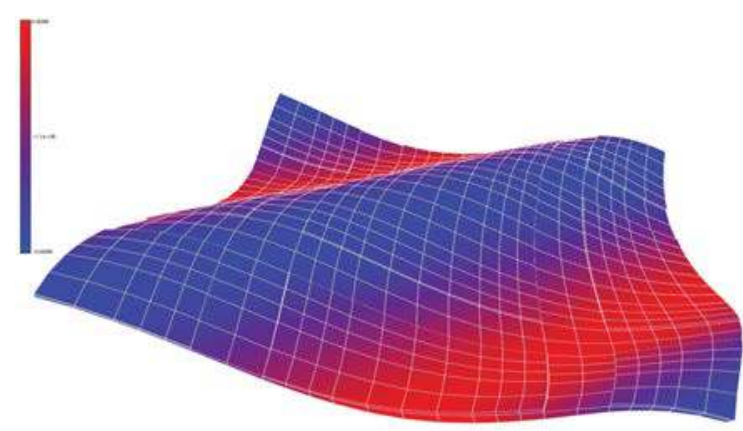

(b)

Fig.5 Deformed mesh for both modes emphasized in fig. $4 \mathrm{~b}$ (isovalues correspond to displacement along $x_{1}$ ), and assumed to correspond to (a) beginning of the stop-band (b) end of the stopband

\section{TRANDUCER FABRICATION AND CHARACTERIZATION}

Once these first computations achieved, its mean to verify the possibility to build such a device and to try and characterize its operation. A process flow chart has been developed in that matter yielding two mask levels to first achieve the 2D periodic $\mathrm{SiO}_{2}$ spacers allowing for insulating the $\mathrm{LiNbO}_{3}$ surface from the connection strips. We assume that the resulting parasitic capacitance will not hide the piezoelectric excitation taking advantage of the strong dielectric constant of the lithium niobate forcing the electric field in the substrate (the outer field being neglected as at least ten times less intense than the one in the substrate). Figure 6 briefly resume this technology fabrication process, superposing the different mask layers to provide the reader a rough idea of the overall apsect of the final device, which corresponds to a one port transducer structures close to the $2 \mathrm{D}$ periodic structure considered in the previous simulation. 


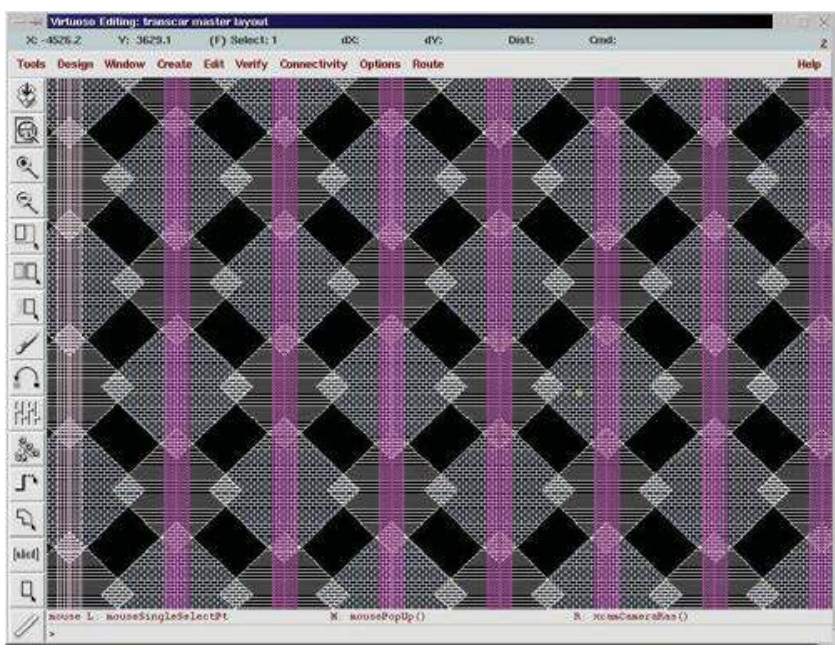

Fig. 6 View of the different mask levels. The large pads corresponds to $\mathrm{SiO}_{2}$ insulators enabling the 2D connection

Figure 7 shows a photo of the 4" $\mathrm{Y}+128^{\circ} \mathrm{LiNbO}_{3}$ wafer ready to be tested on our Suss Microtec tip probing bench. The process was achieved successfully as the mechanical period $(25 \mu \mathrm{m})$ allowed for an easy alignment of the device patterns.

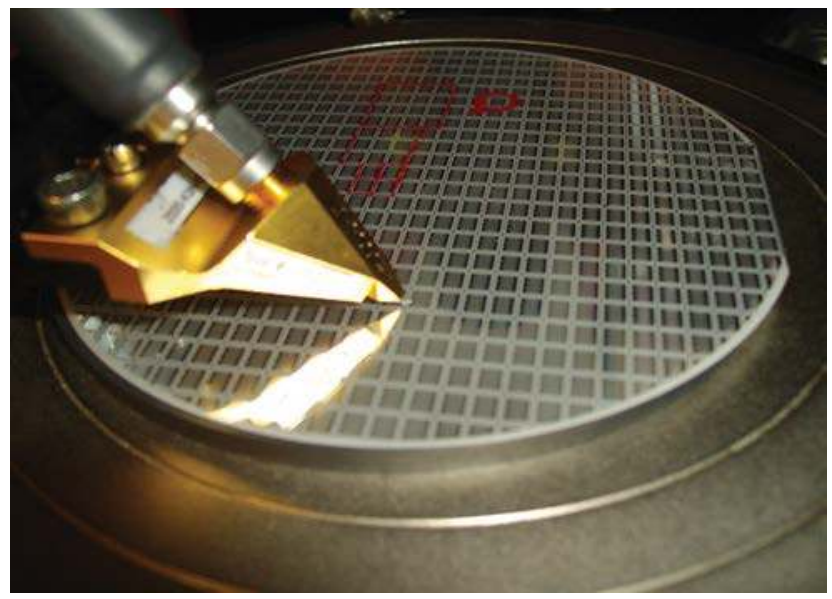

Fig. 7 Photo of the test wafer on the tip prober (Süss Microtec)

The admittance of the device has been measured and is reported in fig.8. A first wide band spectrum shows two principal contributions near $100 \mathrm{MHz}$ and a significant amont of contributions assumed to correspond to plate modes propagating back and forth the substrate surfaces wih modulates the longitudinal bulk wave radiated form the surface near $200 \mathrm{MHz}$ (wave velocity in the vicinity of $7000 \mathrm{~m} \cdot \mathrm{s}^{-1}$ as expected for Lithium Niobate), and even a small contribution near $220 \mathrm{MHz}$. At a glance, the correspondance with the harmonic conductance reported in fig.4a is rather convincing about the fact that the device operates as theoretically announced. When focusing on the low frequency contributions, one can see that they are composed of two principal wide band contributions modulated by resonance peaks, expected to correspond to wave reflection on the surrounding devices. Whatever the origin of this effect, this measurement proofs that the device actually can generate waves with clear electrical contribution on the device admittance and that quite sharp resonance can be expected, although the operation frequency remains rather low (near $100 \mathrm{MHz}$ ), correpodning rather fairly to theoretical predictions. 


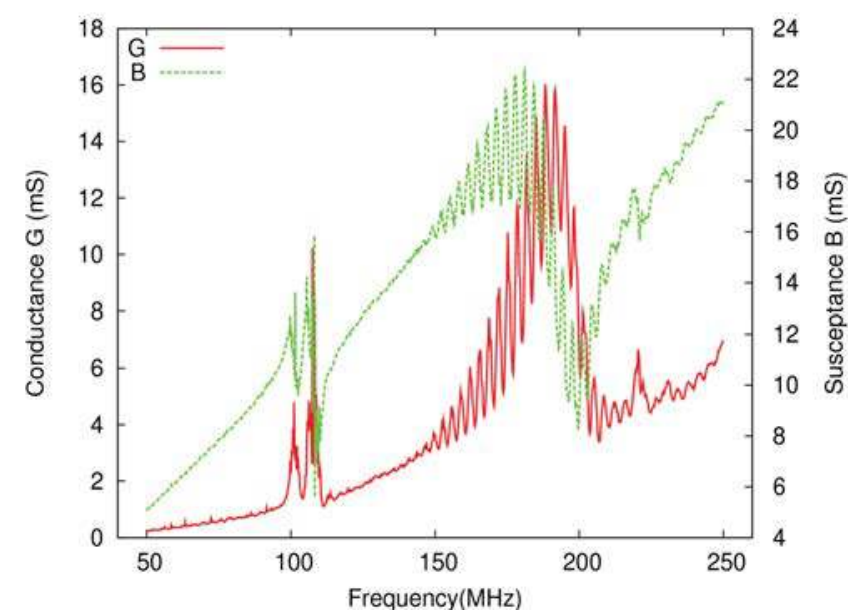

(a)

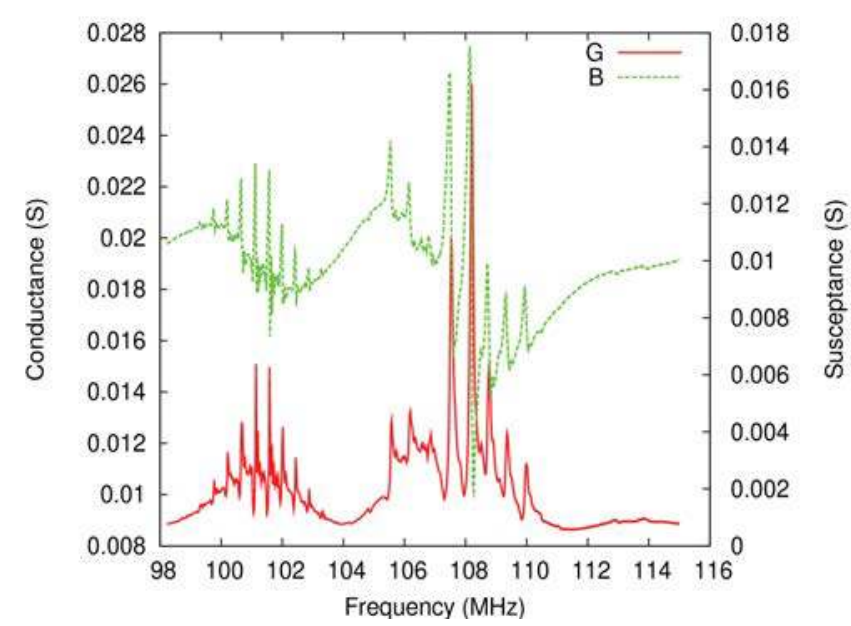

(b)

Fig.8 Experimentally measured admittance of one of the single-port resontor shown in fig.7 (a) wide band spectrum showing two modes near $100 \mathrm{MHz}$ and a strong bulk wave contribution near $200 \mathrm{MHz}$ (b) focus on the two principal mode contributions, revealing a complexe resonance structure due to reflection of the neighbour devices

\section{CONCLUSION}

In this work, we propose a new 2D bi-periodic transducer fabricated on single crystal susbtrates allowng for complex wave polarization excitation. The structure has been theoretically analysed using a mixed finite element/boundary element approach, showing numerous acoustic-electric contribution on the harmonic admittance for quartz. An experimental device then has been built on $\mathrm{LiNb} 03$ using $\mathrm{SiO} 2$ spacers for 2D interconnection purpose. The device has been tested successfully, allowing for a first demonstration of the transuction principle. More work now will be achieve to better understand the transducer operation and to propose actual application of this $2 \mathrm{D}$ transducer.

\section{REFERENCES}

[1] R. M. White, F. W.Votlmer, "Direct piezoelectric coupling to surface elastic waves", Applied Physic Letters 7, 314-316 (1965)

[2] S. Sherrit, B.K. Mukherjee, Proc. of the IEEE Ultrasonics Symp., Sendai, 1, 633 (1998)

[3] S. Ballandras, A. Reinhardt, V. Laude, A. Soufyane, S. Camou, W. Daniau, T. Pastureaud, W. Steichen, R. Lardat, M. Solal, P. Ventura, J. Appl. Phys., Vol. 96, n²12, pp. 7731-7741, 2004

[4] S.Ballandras, M.Wilm, P.F. Edoa, V. Laude, A. Soufyane, W. Steichen, R. Lardat, , J. of Appl. Phys., 93, 702 (2003)

[5] P. Langlet, A.C. Mladky-Hension, J.N. Decarpigny, S. de Physique IV, Colloque C1, 2 (1992).

[6] D.P. Morgan, Surface Wave Devices for Signal processing, Studies in Electrical Eng. 19, Elsevier Ed., 1985 